\title{
Changes in Intracranial Pressure and Arterial Blood Pressure Following Electric Stimulation to Restricted Regions in the Cat Brainstem
}

\author{
Shiushi Matsuura, Miyuki Kuno, Toshiya Yasunami, \\ and Masanobu MAEDA* \\ Department of Physiology and * Department of Neurosurgery, \\ Osaka City University Medical School, \\ Abeno-ku, Osaka, 545 Japan
}

\begin{abstract}
The brainstem of anesthetized cats was electrically stimulated to examine the changes in the intracranial pressure (ICP). There were pressor and depressor sites, which preferentially produced an immediate increase and decrease in ICP in association with the arterial pressor and depressor responses, respectively. A preferential increase in ICP was also observed by stimulation of some depressor sites. The stimulus-induced ICP responses were usually different from the secondary ICP changes due to nonneurogenic alteration of arterial blood pressure (BP) as evoked by arterial bleeding and infusion of saline solution; the stimulus-induced increase in ICP was greatly enhanced when the stimulation to the pressor sites was applied at lowered BP levels and at moderately elevated ICP levels. In addition, when a gradual elevation in ICP was spontaneously observed with the lowering of the BP level, the pressor site-induced increase in ICP exceeded $70-100 \mathrm{mmHg}$ at the peak plateau-like waves, regardless of the magnitude of accompanying arterial pressor response. We propose that the stimulus-induced ICP responses cannot be explained merely by the metabolic changes, the decreased intracranial compliance, and the secondary transmural action on the intracranial space of the arterial pressor and depressor responses. A neurogenic mechanism that directly affects intracranial blood diameter may be involved in the ICP responses, especially those observed at a lower BP level, in addition to extracranic action of sympathetic and parasympathetic nerve activities.
\end{abstract}

Key words: intracranial pressure, pressor response, depressor response, brainstem stimulation, neurogenic vasodilation.

Cerebral blood flow (CBF) remains relatively constant via compensatory vasoconstriction or dilation of cerebral blood vessels due to autoregulation in the presence of hemodynamic changes such as an increase or a decrease in BP (KonTos

Received for publication May 22, 1986 
et al., 1978; BAumbaCH and Heistad, 1983). However, as vascular changes occur within a rigid skull, it is reasonable to assume that the ICP is influenced by a change in blood volume in intracranial vascular beds and alteration in compliance of intracranial content (LANGFITT et al., 1965; LUNDBERG et al., 1968). Changes in pulsatile fluctuation of ICP due to respiratory and cardiac rhythms and other origins related to slow arterial pressure fluctuations (PETTOROss et al., 1978) also have to be considered. On the contrary, moderate and large increases in ICP influence arterial blood pressure as well as cardiac and respiratory rhythms due to changes in sympathetic and parasympathetic outflows (MATSUURA et al., 1984). Furthermore, ROSNER and BECKER (1984) suggested that changes in ICP prior to and during plateau waves, which are characterized by spontaneous and rapid elevations in ICP rising above moderately elevated baseline levels, are dependent on systemic circulatory events, as has been evident under certain circumstances in humans and laboratory animals (LUNDBERG et al., 1968).

Electric stimulation applied to restricted areas in the brainstem also induces arterial pressor and depressor responses via neural activites associated with or without influences on cerebral circulation (LANGFITT and KASSELL, 1968; DoBA and ReIS, 1972; Dampney et al., 1979; GoADSBy et al., 1983). Although it remains unknown whether pressor and depressor areas in the brainstem alter the ICP in relation to changes in $\mathrm{BP}$, we investigated the responses of ICP and $\mathrm{BP}$ induced by local stimualtion of pressor and depressor sites in the brainstem to examine how the brainstem activity modifies ICP by the stimulus-induced activation of the sites.

\section{METHODS}

Thirty-five adult cats of either sex each weighing $2-4.5 \mathrm{~kg}$ were anesthetized with sodium pentobarbitone $(35 \mathrm{mg} / \mathrm{kg}$, i.p.). Arterial blood pressure was measured with a straingauge electric transducer (Nihon Kohden, MPU-0.5A) attached to one of femoral and axillary arterial catheters, the tips of which were introduced into the thoracic aorta; the other remaining catheter was used to inject saline or obtain blood samples. Another polyethylene catheter was inserted into the superficial vein of the foreleg to inject anesthetics and other drugs. The ICP was measured with a straingauge transducer attached to a catheter introduced into the right lateral ventricle or to a small balloon placed in the intracranial space through a parietal craniotomy. The opening in the bone was tightly sealed with dental cement. Electroencephalogram (EEG) was recorded using a screw electrode placed in the left parietal region of the skull. The CBF was monitored by a needle type of thermoelectric element (Shin-ei, WN-501) inserted into the left parietal lobe of the brain and connected to a flow meter (Shin-ei, Shincorder CTE-202). The $\mathrm{CO}_{2}$ level of expired gases was maintained between $3-4 \%$ using an infrared $\mathrm{CO}_{2}$ analyzer (Horiba, MCD-1). The ICP, BP, EEG, CBF, and $\mathrm{CO}_{2}$ were recorded on a polygraph pen recorder (Nihon Kohden, RM 85 or RM 6000). Rectal temperature was monitored and maintained in a range of $35.5 \pm 1.5^{\circ} \mathrm{C}$. 
The head of the cat was fixed in a stereotaxic frame. The stimulating coaxial electrode consisted of a stainless steel barrel and a tungsten core insulated except for the tips. The distance between the core and the circumferential barrel was about $0.5 \mathrm{~mm}$. A burr hole was drilled in the occipital bone and a small incision less than the outer diameter of the electrode was made using an injection needle in the dura mater. The electrode was advanced in steps of $0.5-1.0 \mathrm{~mm}$ with a micromanipulator from the dorsocaudal to the ventrorostral direction at an angle of $54^{\circ}$ or vertically to the Horsley-Clark horizontal plane. The stimulation was given with a train of square cathodal pulses (duration, $0.4 \mathrm{~ms}$; frequency, $40 \mathrm{~Hz}$ ). When a leakage and the outflow of CSF or sucking of the air were observed from the incision of the dura mater around the inserted electrode, the area of incision around the electrode was tightly sealed using dental cement. If necessary, another elecrode inserted through a different tract was used for the following series of stimulation. In some animals, distortion of the brainstem was conducted by inflating the balloon placed in the intracranial space or by moving a stainless steel rod (diameter, $0.8 \mathrm{~mm}$ ), which was inserted into the lateral part of the brainstem to the cranial base, along the longitudinal axis (within about $2 \mathrm{~mm}$ ). When respiratory arrest was observed by stimulation, the experiment was conducted under artificial ventilation with the use of pancuronium bromide. Additional doses of anesthetics (pentobarbitone: 3$5 \mathrm{mg} / \mathrm{kg}$ in 25 cats and $\alpha$-chloralose: $5-10 \mathrm{mg} / \mathrm{kg}$ in 10 cats) and muscle relaxant were given, at about $1-1.5 \mathrm{~h}$-intervals.

At the end of each experiment, the brain was removed and fixed in $10 \%$ formaldehyde saline for histological studies of the locations stimulated. The locations were determined with respect to the obex, middle line, outlet of V, VI, VIII, X, and XII cranial nerves, and dorsal and ventral surfaces, referring to the atlas of SNIDER and NIEMER (1961).

\section{RESULTS}

Responses in ICP and BP to the stimulation of depressor and pressor areas in the brainstem and their brainstem distribution

Electric stimulation was applied on different points over the brainstem areas extended rostrocaudally, from $\mathrm{p} 2$ to $\mathrm{p} 14$. Stimulation of many sites of the pressor area produced an increase in ICP (Fig. 1A). Such increase in the response in ICP associated with an arterial pressor response (Fig. 2, open circles) was consistently observed by the stimulation of sites dorsolateral to the paramedian region (Fig. 2A-D), the ventrolateral sites (Fig. 2E-F) of the medulla and the fastigial nucleus (Fig. 2A and B) in many cats. Stimulation of some midline sites of the brainstem (Fig. 2B) also produced arterial pressor responses associated with a rise in ICP. In the paramedian region near the midline of the brainstem (Fig. 2A-C, closed circles), depressor sites responsible for an increase in ICP and arterial depressor response (Fig. 1B) were also consistently observed in many cats. In the depressor area caudal to the obex (Fig. $2 \mathrm{~F}$, open triangles), arterial depressor responses that occurred in 

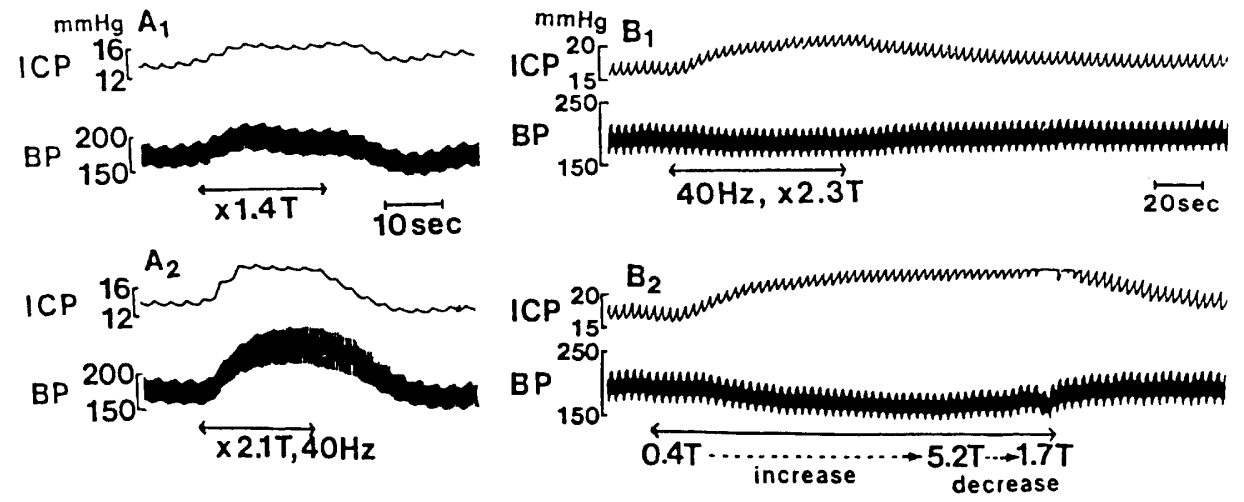

Fig. 1. Changes in ICP and BP produced by electric stimulation of the brainstem. $A_{1}-A_{2}$ and $B_{1}-B_{2}$ are the responses to different intensities of stimulation to two different sites (Fig. 2a and b) of the cat ventilated artificially. Durations of stimulation are shown by horizontal arrows in this and the following figures, and stimulus intensities are expressed in terms of the smallest intensity (threshold intensity $=1 \mathrm{~T}$ ) to evoke a response.

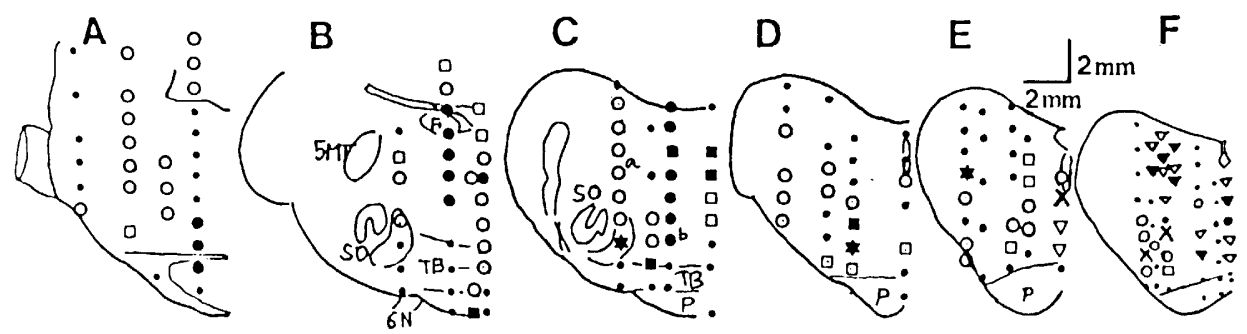

Fig. 2. Schematic drawing showing the points electrically stimulated in the serially sectioned block of brainstem (at $54^{\circ}$ to the Horsley-Clark horizontal plane). Each block was $2-3 \mathrm{~mm}$ thick, and $10,8,6,4$, and $1 \mathrm{~mm}$ rostral and $2 \mathrm{~mm}$ caudal to the obex at the level of the floor of the 4th ventricle for A-F, respectively. Each stimulation was applied in the left or right side, but the results are represented on the half blocks. Different symbols show the sites which produced a decrease in ICP associated with an increase $(\times)$, a decrease $(\nabla)$, and no change $(*)$ in BP; an increase in ICP with an increase (O), a decrease (๑), and no change in BP $(\square)$, an increase only in BP $(\boldsymbol{\square})$; a decrease only in BP $(\nabla)$; no responses in ICP and BP $(\bullet)$. These results were obtained from 201 sites stimulated in 8 cats.

association with a decrease in ICP were usually observed in many cats, though some sites in the area produced no apparent change in ICP (Fig. $2 \mathrm{~F}$, closed triangles). These responses were reproducible in many cats.

The responses in BP and ICP usually started within a few s after initiation of stimulation and reached a peak within $10 \mathrm{~s}$. In addition, when the arterial pressor response was produced with an increase in ICP, the time course in the rising phase 

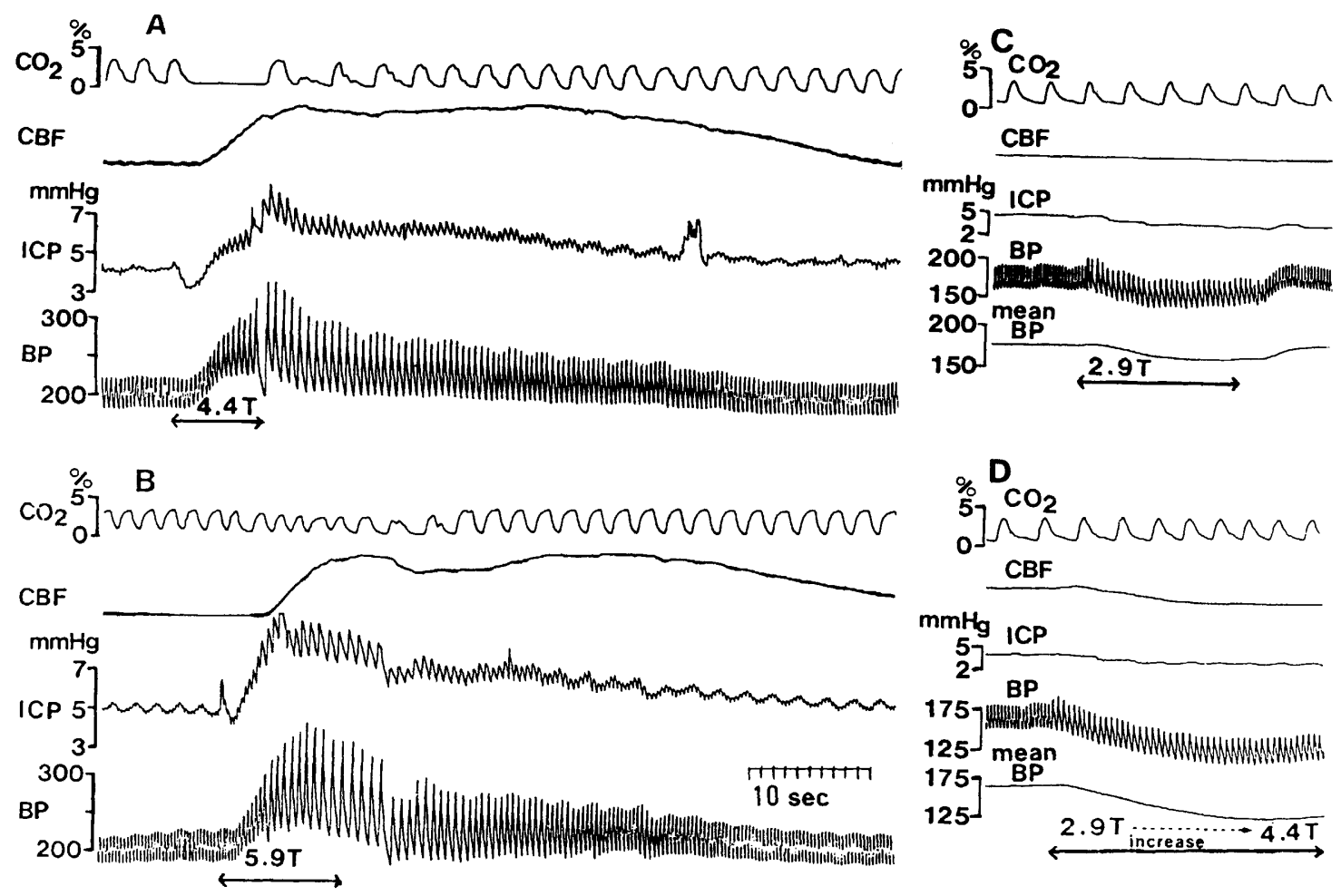

Fig. 3. Changes in BP, ICP, and respiratory rhythm produced by electric stimulation of the brainstem in the spontaneously ventilated cat. A and B are the responses to stimulations of two different sites in the pressor area. $C$ and $D$ are the responses to stimulation of a site in the depressor area. In each record of CBF, upward deflection shows an increase and downward, a decrease in the flow. Stimulus intensity and the duration are shown in each record. 
was more rapid in the ICP pressor response than in the arterial pressor response in most trials. At some pressor sites, no apparent change (Fig. 2B-D, closed squares) or a decrease (Fig. 2E and F, crosses) was produced in ICP even when the arterial pressor response exceeded about $50 \mathrm{mmHg}$. The decreased ICP in association with the arterial pressor response were observed in 3 cats, but not in the other 15 cats in which the same brainstem area was stimulated. In 4 out of 19 cats there was no apparent change in ICP associated with the arterial pressor response by stimulation. A decrease in ICP without a change in BP (Fig. 2, stars) was observed only in a few cats.

Stimulation of some sites evoked respiratory arrest (Fig. 3A), an increase in respiratory rhythm (Fig. 3B and D) or somatic muscle contraction (body movement), in association with changes in BP and ICP in the cats spontaneously respirated, though the stimulation also produced changes in BP and ICP when the cat was immobilized with a muscle relaxant and artificially ventilated. Figure 2 shows the data obtained from 8 animals which did not show leakage of CSF, apparent body movement or respiratory arrest, thereby being free from possible secondary effect of stimulation attributable to pressure changes of mechanical origins. In addition, the same intensity of pulses $(120 \mu \mathrm{A})$ was used for each stimulation in Fig. 2. When biphasic responses, i.e., an increase followed by a decrease, was observed in BP and ICP during stimulation, the latter component of the biphasic responses dominated over the former, when the intensity of stimulus was gradually increased. Such site was classified by the latter component. Otherwise, the response for the site was classified by the component with larger magnitude.

In some cases, a small stepwise movement of the electrode produced a change in BP and/or ICP, in the absence of electric stimulation to the sites. Most of the sites also produced stimulus-induced responses in BP and ICP, similar to the changes seen with advancement of the electrode.

In 10 cats anesthetized with $\alpha$-chloralose, the responses in ICP and BP were the same as those in the cats anesthetized with sodium pentobarbitone. A quantitative analysis of the response between the cats anesthetized with sodium pentobarbitone and $\alpha$-chloralose was not made.

Differences between the ICP responses evoked by stimulation and those ascribed to hemodynamic origin such as bleeding and infusion of saline into the aorta

Changes in BP by rapid hemorrhage or infusion of a saline solution also produced a decrease or an increase in ICP. Then, magnitudes of ICP changes by stimulating pressor and depressor sites were respectively compared with those provoked by the infusion of saline and hemorrhage in 5 cats. The purpose was to examine whether the origin of stimulus-induced ICP responses is ascribed to the hemodynamic changes alone or not. The ICP changes in response to stimulation were different in magnitude from those produced by hemodynamic changes, even when the BP change was equalized in the amplitude by controlling either stimulus 

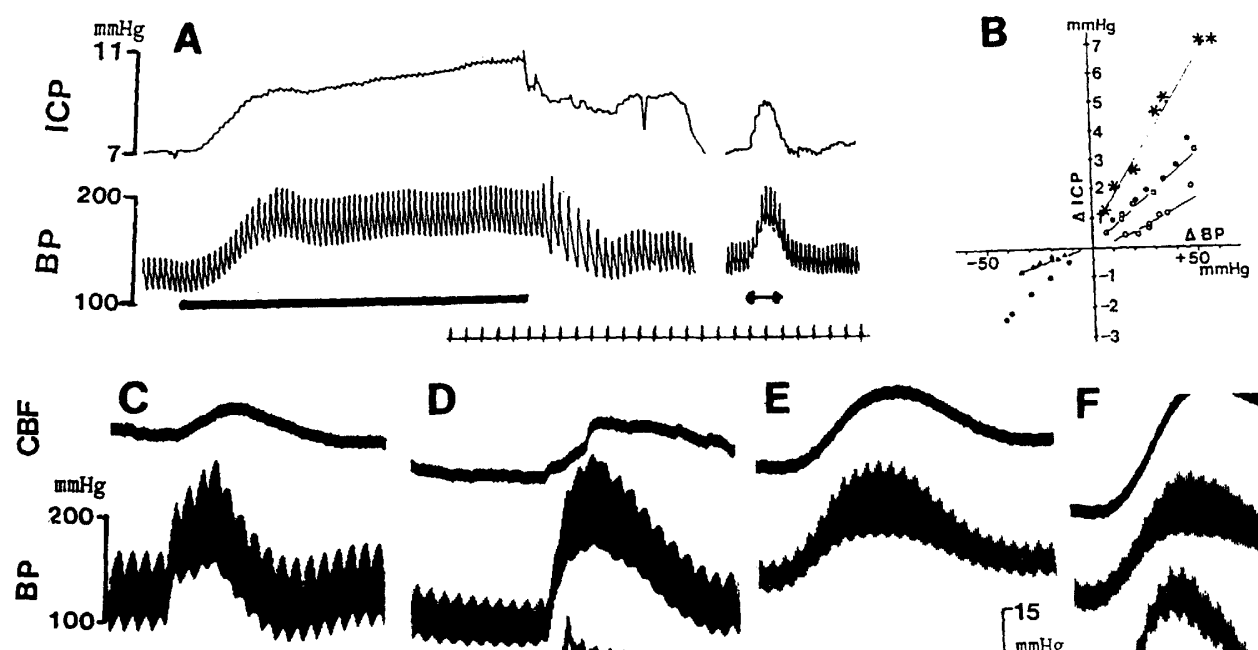

옹
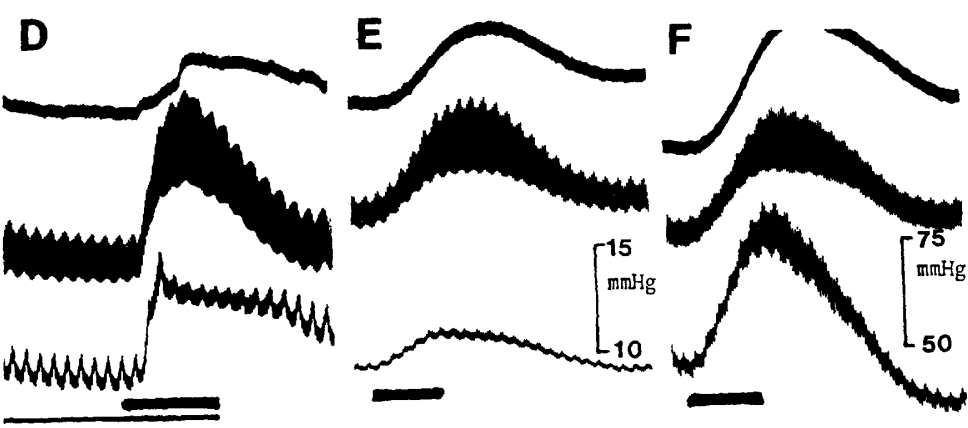

$10]$

Fig. 4. Comparison between the responses evoked by electric stimulation and the responses to bleeding or infusion of saline solution into the aorta, and enhancement of the stimulus-induced ICP responses at lowered BP and at increased ICP level. A, sample of the stimulus-induced ICP response and the response by infusing saline solution into the artery $(2 \mathrm{ml}$; arrow) which produced the same amplitudes of pressor response. The unit of time scale is $1 \mathrm{~s}$. B, magnitude in the change of ICP is shown against the magnitude in the change of $\mathrm{BP}$ for the responses to different amounts of bleeding and infusion (closed circles) and the responses to different intensities of stimulation at four different sites (stars and open symbols). The baseline BP was $100-$ $105 \mathrm{mmHg}$ for closed symbols and $120-125 \mathrm{mmHg}$ for open symbols. $\mathrm{C}$ and $\mathrm{D}$, stimulus-induced responses before (C) and during the lowered levels of BP (D) by stimulating the depressor site (thin bar). The stimulation of the depressor site was begun about $30 \mathrm{~s}$ before the record of $\mathrm{D}$. $E$ and $F$, stimulus-induced responses before (E) and during the elevated ICP and the associated lowering of BP by infusing saline into lateral ventricle $(F)$. The thick bars show the duration of stimulation to the pressor sites. The unit of time 
intensity or amount of volume loading (Fig. 4A). Therefore, when the amplitudes of increased ICP were plotted against the amplitudes of increased BP, there were differences between the response to hemodynamic changes and those to activation of pressor sites (Fig. 4B).

Effects on stimulus-induced $B P$ and ICP responses of prestimulus levels of $B P$ and ICP and of a distortion of the brainstem

An alteration in cardiovascular activity and a change in cerebral blood flow were produced by an increase in ICP and the axial brainstem distortion as well as the effects of hypotension on cardiovascular activity. Therefore, an increase in the prestimulus ICP level, brainstem distortion and/or hypotension were induced to examine the effects on the stimulus-induced ICP responses in 7 cats. When the prestimulus BP level (mean BP) was decreased by stimulating the depressor site, for example, to about $100 \mathrm{mmHg}$, an apparent increase was observed in the amplitude of ICP response by stimulating the pressor site (Fig. 4C and D). Moderately elevated levels of ICP, which frequently induced an associated decrease in BP level, produced a much larger increase in the stimulus-induced ICP response such as 25 $50 \mathrm{mmHg}$ in the response amplitude (Fig. 4E and F). The increase in the ICP response cannot be explained by the change of intracranial compliance alone, since the lowered BP level without an increase in ICP level also produced an enhancement in the stimulus-induced ICP response. At the control levels of BP (mean BP; about $110-155 \mathrm{mmHg}$ ) and ICP (without about $18 \mathrm{mmHg}$ ), amplitudes of the stimulusinduced ICP responses were usually less than about $3.5 \mathrm{mmHg}$, and responses larger than $5 \mathrm{mmHg}$ were not observed.

After a marked elevation of ICP by inflation of an intracranial balloon and/or the brainstem distortion, the stimulus-induced ICP response and the pressor response were sometimes followed by an apparent gradual elevation of ICP after the end of stimulation (Fig. 5B). We also noted small spontaneous changes in BP and ICP during the course of most experiments without the inflation of a balloon to elevate ICP and the brainstem distortion, but the changes in BP and ICP levels remained within a small range of about 20 and $5 \mathrm{mmHg}$, respectively.

Contrary to the gradually elevated and then sustained baseline level of ICP (Fig. 5B and C), BP returned either to the prestimulus control level or slightly undershot to a lower level. At the elevated ICP level and the lowered BP level, the stimulus effects were quite different from those observed before the elevation of ICP. We noted the occurrence of a marked plateau-like increase in the ICP which are sometimes observed clinically in ICP. The plateau-like wave in ICP preceded the start of the arterial pressor response or occurred in the absence of a change in BP, as is evident in the response to a low intensity of stimulation (Fig. 5C). However, in the control response before the change of the baseline levels of ICP and BP, stimulation to the site never produced the response in ICP without an arterial pressor response.

In contrast to the effects of a moderately increased ICP level which produced no apparent change in EEG, repeated applications of marked increases (each 

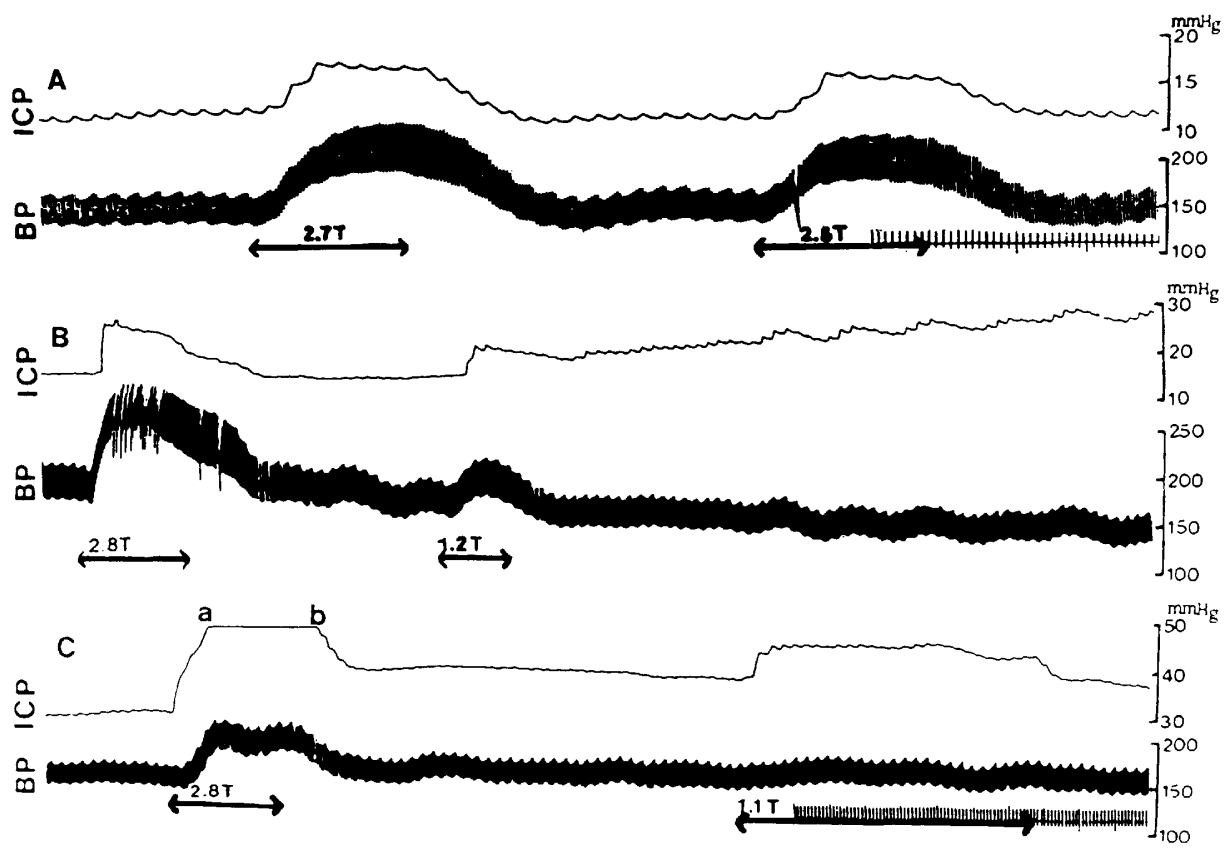

Fig. 5. Changes in the stimulus-induced responses after inflation of the supratentorial balloon and brainstem distortion. A, responses in ICP and BP to stimulation to a site in the pressor area before inflation of the balloon. B, stimulus-induced response taken about $20 \mathrm{~min}$ after the inflation and the following gradual elevation of ICP which was associated with lowering of BP. The balloon was inflated several times by injecting various amounts of saline (within $1.5 \mathrm{ml}$ ). Each injection was conducted after the recovery of EEG waves and/or BP, if slowing in EEG waves and/or an increase in BP had occurred in association with the preceding inflation and the increase in ICP. C, recordings continue from $\mathrm{B}$, but were interrupted about $5 \mathrm{~min}$ between $B$ and $C$. The ICP response saturated between $a$ and $b$ because it exceeded the range of the recording system. The unit of time scale shows $1 \mathrm{~s}$.

duration: less than 3-5 min) in ICP and the brainstem distortion, which induced Cushing responses and slowing of EEG waves, produced disappearance of stimulusinduced responses in ICP and BP. The arterial depressor response and the associated ICP responses seemed to be more easily affected than the arterial pressor response and the associated increase in ICP. Recovery of the stimulus-induced responses first occurred within $20-40 \mathrm{~min}$, but no recovery occurred with further repeated applications of such maneuvers, which indicates a progressive deterioration of brainstem neurons responsible for the responses.

\section{DISCUSSION}

We found that ICP responses were induced by stimulating restricted brainstem 
sites as well as the arterial pressor and depressor responses and that the ICP responses cannot be explained merely by the secondary, transmural action of the BP change evoked by the activation of brainstem sites. Though the net volume changes in any of the intracranial contents within the rigid skull, which are induced by vascular autoregulation of cerebral blood vessels due to metabolic changes (LANGFITT et al., 1965), chemical substances like angiotensin (BARBELLA et al., 1983), and any other causes (LUNDGERG et al., 1968), produce a change in ICP, the rapid changes and reversibility of ICP and the enhancement of the stimulus-induced increase in ICP at the lowered BP level indicate that the ICP responses are primarily caused by alteration in blood volume due to neurogenic dilation and constriction of intracranial vessels, thereby permitting some of the systemic blood pressure to affect the geometry of the vascular bed, as is the case with a possible origin of the plateau waves of ICP (LUNDBERG et al., 1968). The primary neurogenic response of intracranial blood vessels was also proposed in case of electrical stimulation of fastigial nucleus (NAKAI et al., 1983) parasympathetic nerve stimulation (D'ALECY and Rose, 1977) and sympathetic nerve stimulation (WEI et al., 1975; AUER et al., 1983; BAUMBACH and HEISTAD, 1983). The neurogenic control mechanism in case of sympathetic nerve stimulation operated to affect CBF more effectively during hypercapnia (BusiJA and HeISTAD, 1984) and at increased BP levels (Kontos et al., 1978). Neurogenic influence on cerebral vascular bed was also proposed in case of stimulation of locus coeruleus (GoADSBY et al., 1983).

Though various ICP responses were observed in relation to the arterial pressor and depressor responses to stimulation of different brainstem sites, the stimulusinduced responses consistently and easily observed in many cats were an increase in ICP associated with the arterial pressor response and a decrease in ICP associated with the arterial depressor response. An ICP increase in association with arterial depressor response was also produced rather more frequently than the other combination of arterial and ICP responses to stimulation. These consistently and more frequently observed responses in many cats may be preferentially assumed to have a primary functional meaning on the regulation or modification of ICP as well as BP. The mutual relationship and interference among ICP, BP, and activities of the pressor and depressor sites are explained by the series of events supposed to occur following activation of the pressor or depressor sites, since changes in cardiovascular activity and CBF were observed by an increase in ICP (LITTLE and Öberg, 1981; Sadoshima et al., 1981; MatsuUra et al., 1984; Nagao et al., 1984) and an alteration in BP (Kontos et al., 1978; KumAdA et al., 1979). A schematic diagram in Fig. 6 illustrates that the increased prestimulus ICP levels, the lowered $\mathrm{BP}$ and other factors like intracranial compliance always interfere with the stimulusinduced effects of pressor sites on ICP and BP by changing their sensitivity through the mutual facilitatory and inhibitory actions, as is shown in the figure.

The occurrence of marked plateau-like waves of ICP at the gradually elevated levels of ICP and the lowered levels of BP following repetition of stimulations after artificial increases in ICP and/or brainstem distortion is explained mainly by an 


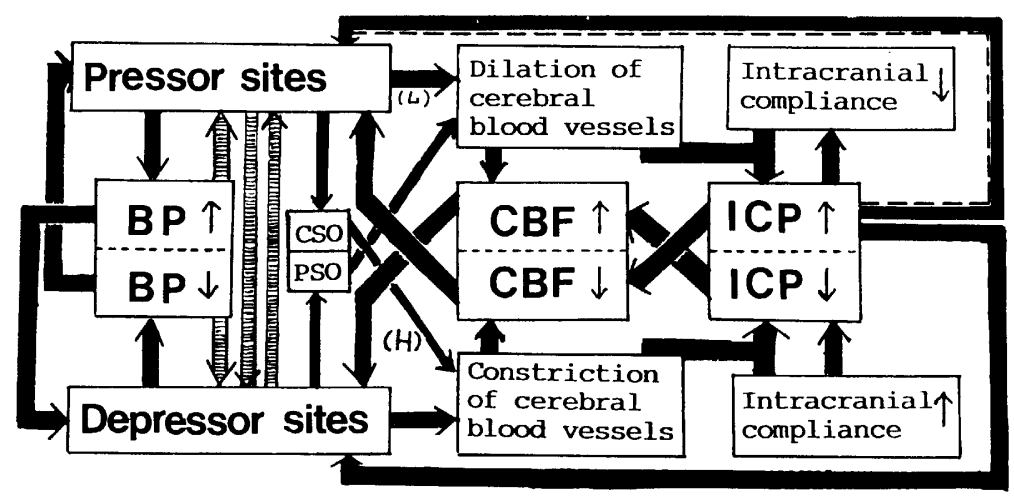

Fig. 6. Schematic illustration of neurogenic effects of activation of pressor and depressor sites on BP and ICP, and the mutual interferences among changes in ICP, BP, and the brainstem activities. Each black arrow indicates an excitatory or facilitatory action, and shaded arrows inhibitory or suppressing action. The arrow attached with a dotted line indicates a facilitatory action at markedly increased levels of ICP but a suppressive action at the moderately increased levels. CSO and PSO show cervical sympathetic nerve outflow and cranial parasympathetic nerve outflow to cerebral blood vessels, respectively. The $\mathrm{H}$ within parentheses indicates that increased CSO works more effectively to control CBF at increased BP levels (Kontos et al., 1978) and during hypercapnia (BUSIJA and HeISTAD, 1984). The $\mathrm{L}$ within parentheses was proposed to work effectively at the lowered BP from the present observation.

increase in blood volume due to possible neurogenic dilation of cerebrovascular beds by stimulation, since plateau-like waves occurred with no apparent arterial pressor response by a low intensity of stimulation. The observations support the explanation by ROSNER and BECKER (1984) that these plateau waves occur as the result of intact or mostly intact cerebral blood vessels responding to changes in cerebral perfusion pressure that induce an increase in cerebral blood volume.

All these observations taken together suggest that the activation of different sites of the brainstem by stimulation produces a change in ICP, and that the changes in ICP occur in a direct and a dissociated relation to actions on the extracranial vascular bed and to the change in BP. We conclude that changes of intracranial blood volume responsible for an increase in ICP can be induced by activation of neural elements in the brainstem, which directly affects the diameter of the cerebrovascular vessels. In addition, the ICP pressor response is proposed to be more easily induced at a moderately elevated ICP level and a lowered BP level.

This study was supported in part by a Grant-in-Aid for Scientific Research from the Ministry of Education, Science and Culture of Japan (59480108). 


\section{REFERENCES}

AuER, L. M., Edvinsson, L., and Johansson, B. B. (1983) Effect of sympathetic stimulation and adrenoceptor blockade on pial arterial and venous calibre and on intracranial pressure in the cat. Acta Physiol. Scand,, 119: 213-217.

Barbella, Y. R., Keil, L. C., Wurpel, J. N. D., and Severs, W. B. (1983) Cerebrospinal fluid pressure during cerebroventricular infusion of angiotensin and vasopressin. Exp. Neurol., 82: 325-334.

Baumbach, G. L. and Heistad, D. D. (1983) Effects of sympathetic stimulation and changes in arterial pressure on segmental resistance of cerebral vessels in rabbits and cats. Circ. Res., 52: 527-533.

Busija, D. W. and Heistad, D. D. (1984) Effects of activation of sympathetic nerves on cerebral blood flow during hypercapnia in cats and rabbits. J. Physiol. (Lond.), 347: 35-45.

D'AleCY, L. G. and Rose, C. J. (1977) Parasympathetic cholinergic control of cerebral blood flow in dogs. Circ. Res., 41: 324-331.

Dampney, R. A. L., Kumada, M., and Reis, D. J. (1979) Central neural mechanisms of the cerebral ischemic response. Characterization, effects of brainstem and cranial nerve transections, and simulation by electrical stimulation of restricted regions of medulla oblongata in rabbits. Circ. Res., 45: 48-62.

Doba, N. and Reis, D. J. (1972) Localization within the lower brainstem of a receptive area mediating the pressor response to increased intracranial pressure (the Cushing response). Brain Res., 477: 487-491.

Goadsby, P. J., Lambert, G. A., and Lance, J. W. (1983) Effects of locus coeruleus stimulation on carotid vascular resistance in the cat. Brain Res., 278: 175-183.

Kontos, H. A., Wei, E. P., Navari, R. M., Levasseur, J. E., Rosenblum, W. I., and Patterson, J. L. (1978) Responses of cerebral arteries and arterioles to acute hypotension and hypertension. Am. J. Physiol., 234: H371-H383.

Kumada, M., Dampney, R. A. L., and Reis, D. J. (1979) Profound hypotension and abolition of the vasomotor component of the cerebral ischemic response produced by restricted lesions of medulla oblongata in rabbit. Relationship to the so-called tonic vasomotor center. Circ. Res., 45: 63-70.

LANGFITT, T. W. and Kassell, N. F. (1968) Cerebral vasodilatation produced by brainstem stimulation: Neurogenic control vs. autoregulation. Am. J. Physiol., 215: 90-97.

Langfitt, T. W., Weinstein, J. D., and Kassell, N. F. (1965) Cerebral vasomotor paralysis produced by intracranial hypertension. Neurology, 15: 622-641.

LITTLE, R. A. and ÖBERG, B. (1981) Arterial baroreceptor reflex function during elevation of intracranial pressure. Acta Physiol. Scand., 112: 27-32.

LundBerg, N., Cronqvist, S., and KJÄLlquist, $\AA$. (1968) Clinical investigation on interrelations between intracranial pressure and intracranial hemodynamics. Prog. Brain Res., 30: 69-75.

Matsuura, S., SaKamoto, H., Hayashida, Y., and Kuno, M. (1984) Efferent discharges of sympathetic and parasympathetic nerve fibers during increased intracranial pressure in anesthetized cats in the absence and presence of pressor response. Brain Res., 305: 291301.

Nakai, M., Iadecola, C., Ruggiero, D. A., Tucker, L. W., and Reis, D. J. (1983) Electrical stimulation of cerebellar fastigial nucleus increases cerebral cortical blood flow without change in local metabolism: Evidences for an intrinsic system in brain 
for primary vasodilation. Brain Res., 260: 35-49.

Nagao, S., Sunami, N., Tsutsui, T., Honma, Y., Momma, F., Nishiura, T., and Nishimoto, A. (1984) Acute intracranial hypertension and brain-stem blood flow. An experimental study. J. Neurosurg., 60: 566-571.

Pettorossi, V. E., Di Rocco, C., Caldarelli, M., Mancinelli, R., and Velardi, F. (1978) Influences of phasic changes in systemic blood pressure on intracranial pressure. Eur. Neurol., 17: 216-225.

RosNer, M. J. and BECKER, D. P. (1984) Origin and evolution of plateau waves. Experimental observations and a theoretical model. J. Neurosurg., 60: 312-324.

Sadoshima, S., Thames, M., and Heistad, D. (1981) Cerebral blood flow during elevation of intracranial pressure: Role of sympathetic nerves. Am. J. Physiol., 241: H78H84.

SNider, R. S. and Niemer, W. T. (1961) A Stereotaxic Atlas of the Cat Brain, The Univ. Chicago Press, Chicago.

Wei, E. P., Raper, A. J., Kontos, H. A., and Patterson, J. L., Jr. (1975) Determination of response of pial arteries to norepinephrine and sympathetic nerve stimulation. Stroke, 6: $654-658$. 